\title{
Coincidence of COVID-19 epidemic and olfactory dysfunction outbreak in Iran
}

\author{
Seyed Hamidreza Bagheri ${ }^{1}$, Alimohamad Asghari ${ }^{2}$, Mohammad Farhadi ${ }^{1}$, Ahmad Reza Shamshiri ${ }^{3}$, Ali Kabir ${ }^{4}$, \\ Seyed Kamran Kamrava ${ }^{1}$, Maryam Jalessi ${ }^{2}$, Alireza Mohebbi ${ }^{1}$, Rafieh Alizadeh ${ }^{1}$, Ali Asghar Honarmand ${ }^{5}$, \\ Babak Ghalehbaghi*1 ${ }^{\mathbb{D}}$, Alireza Salimi ${ }^{6}$, Fatemeh Dehghani Firouzabadi ${ }^{1}$ \\ Received: 6 May 2020 \\ Published: 15 Jun 2020
}

\section{Abstract}

Background: The occurrence of anosmia/hyposmia during novel Coronavirus disease 2019 (COVID-19) may indicate a relationship between coincidence of olfactory dysfunction and coronavirus disease 2019 (COVID-19). This study aimed to assess the frequency of self-reported anosmia/hyposmia during COVID-19 epidemic in Iran.

Methods: This population-based cross sectional study was performed through an online questionnaire from March 12 to 17, 2020. Cases from all provinces of Iran voluntarily participated in this study. Patients completed a 33-item patient-reported online questionnaire, including smell and taste dysfunction and their comorbidities, along with their basic characteristics and past medical histories. The inclusion criteria were self-reported anosmia/hyposmia during the past 4 weeks, from the start of COVID-19 epidemic in Iran.

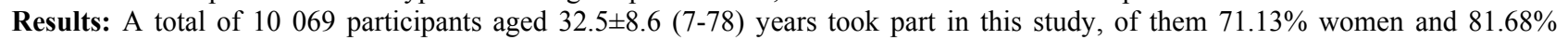
nonsmokers completed the online questionnaire. The correlation between the number of olfactory disorders and reported COVID-19 patients in all provinces up to March 17, 2020 was highly significant (Spearman correlation coefficient $=0.87, \mathrm{P}<0.001$ ). A sudden onset of olfactory dysfunction was reported in $76.24 \%$ of the participations and persistent anosmia in $60.90 \%$ from the start of COVID19 epidemic. In addition, $80.38 \%$ of participants reported concomitant olfactory and gustatory dysfunctions.

Conclusion: An outbreak of olfactory dysfunction occurred in Iran during the COVID-19 epidemic. The exact mechanisms by which anosmia/hyposmia occurred in patients with COVID-19 call for further investigations.

Keywords: Coronavirus, COVID, COVID-19, SARS-CoV-2, Anosmia, Smell, Hyposmia, Dysgeusia, Taste loss, Gustatory, Olfactory, Olfaction, Infection, ENT

Conflicts of Interest: None declared

Funding: None

\section{*This work has been published under CC BY-NC-SA 1.0 license. \\ Copyright $\odot$ Iran University of Medical Sciences}

Cite this article as: Bagheri SH, Asghari A, Farhadi M, Shamshiri AR, Kabir A, Kamrava SK, Jalessi M, Mohebbi A, Alizadeh R, Honarmand AA, Ghalehbaghi B, Salimi A, Dehghani Firouzabadi F. Coincidence of COVID-19 epidemic and olfactory dysfunction outbreak in Iran. Med J Islam Repub Iran. 2020 (15 Jun);34:62. https://doi.org/10.47176/mjiri.34.62

\section{Introduction}

Olfactory dysfunction following the upper respiratory tract infections also named postviral anosmia has been fre-
Corresponding author: Dr Babak Ghalehbaghi, ghalebaghi.b@iums.ac.ir

1. ENT and Head and Neck Research Center and Department, The Five Senses Institute, Iran University of Medical Sciences, Tehran, Iran

2. Skull Base Research Center, The Five Senses Institute, Iran University of Medical Sciences, Tehran, Iran

3. Department of Epidemiology and Biostatistics, School of Public Health, Tehran University of Medical Sciences, Tehran, Iran

4. Minimally Invasive Surgery Research Center, Iran University of Medical Sciences, Tehran, Iran

5. Iran Medical Council, Tehran, Iran

6. Shahid Beheshti University of Medical Sciences, Tehran, Iran $\uparrow$ What is "already known" in this topic:

Since its outbreak, 2019 novel Coronavirus (SARS-CoV-2) has been associated with a spectrum of manifestations, and the full picture of COVID-19 is not yet fully understood. Hence, the various aspects of olfactory dysfunction in COVID-19 should be identified for early detection and isolation of infected individuals.

\section{$\rightarrow$ What this article adds:}

Our findings determined a significant linear correlation between the prevalence of COVID-19 and olfactory dysfunction. In an online survey, $76.24 \%$ of the participations reported a sudden onset of olfactory dysfunction. Findings of this study could lead to the worldwide awareness of researchers to conduct further clinical studies to find any correlation between disease severity and olfactory dysfunction to complete clinical manifestations of COVID-19. 
quently reported in previous studies $(1,2)$. Epithelial damage and central nervous system involvement have been postulated as the probable reasons, however, the exact pathogenesis remains unclear $(2,3)$. Generally, postviral anosmia is more common in middle-aged women (4). Coronaviruses are a large family of viruses that have been presented as the causative agents of different clinical manifestations that range from a common cold to severe and great global public health concerns such as Middle East Respiratory Syndrome (MERS-CoV) and Severe Acute Respiratory Syndrome (SARS-CoV). SARS-Cov2 is a new strain from this family, which has commenced in the world in $2019(5,6)$. The World Health Organization (WHO) has reported 167515 confirmed cases of COVID-19 with 6606 deaths by March 16, 2020 globally (7). As the virus has newly been identified, new reports about the various aspects of the disease are released almost every day during this pandemic $(6,8)$. However, an update on its clinical and laboratory presentations has reported fever, respiratory symptoms, cough, fatigue, myalgia, arthralgia, and breathing difficulties as the common presentations of the confirmed cases (9).

Although olfactory loss had not been mentioned as a presentation of COVID-19 until March 17, 2020, several anecdotal reports were made by Iranian otolaryngologists regarding an outbreak of olfactory loss in social media. The first, albeit was not a peer-reviewed study, was from China reporting a frequency of $5.1 \%$ for smell loss (10). This study was designed to assess the prevalence of olfactory dysfunction in Iran during the COVID-19 pandemic as a preliminary epidemiologic study.

\section{Methods}

\section{Measurement}

An online questionnaire was developed by a board, including otolaryngologists and epidemiologists, from Ear, Nose, Throat and Head and Neck Surgery Research Center of Iran University of Medical Sciences with the cooperation of Iran Medical Council. The software incorporated into the website of Iran Medical Council for corona epidemics (www.corona.ir). The full online questionnaire, which is in Google document format (go.irimc.org/smelltest), contains 33 questions, of which 18 are about any changes in smell or taste and most of the related comorbidities, 13 about basic characteristics of participants (age, date of birth, gender, city of residence, medical history, habitual history, family history), and 2 multiple-choice questions about past medical history (asthma, diabetes, thyroid disease, hypertension, epilepsy, and Parkinson's disease). Participants were asked to report their ability to smell and taste before and during the COVID-19 disease quantitatively via 10point visual analogue scales. Questions about olfactory and gustatory dysfunctions can be seen in the Appendix 1. To check the validity of the data, the questions were cross-validated with each other. For example, if participants mentioned that they had hyposmia but pointed to the zero in the Likert scale in the questionnaire, they were excluded from the analysis. To detect and omit duplicated entries, personal information (age, gender, and location) was compared be- fore data analysis. Furthermore, to exclude incomplete responses to the questionnaire, the records with more than $30 \%$ missing data were deleted.

\section{Study population and clinical outcomes}

This cross sectional study was conducted on 10069 cases in all provinces of Iran from March 12 to 17, 2020. Participants were cases with decreased (hyposmia) or loss of sense of smell (anosmia) during the past 4 weeks, who voluntarily responded to the online questionnaire via the internet (go.irimc.org/smelltest). The inclusion criteria were olfactory dysfunctions in responders within the 4 weeks of the start of COVID-19 epidemic in Iran. Specific groups in the populations were underrepresented because they had less access to the internet, personal computers, or smartphones, or were disable or illiterate to use the internet.

The primary outcome of the study was olfactory dysfunction of the responders at the onset of their problem and its condition at the time of their response to the questionnaire, which was measured by closed questions and scored by Likert scale. Secondary outcomes were clinical manifestations of participants such as flu or cold symptoms before anosmia, headache that needed a pain reliever drug, nasal stiffness, fever and chills, prominent cough, orbital (periorbital) pain, facial fullness and sinus pain, rhinorrhea, dyspnea, nasal irritation, parosmia, nasal pruritus, history of hypothyroidism, otalgia, sneezing (frequent), purulent nasal discharge, history of asthma, cheeks pain, previous sinus surgery or septorhinoplasty, unilateral facial palsy, and a history of hypertension, diabetes mellitus, and hyperthyroidism.

\section{Ethical approval}

Informed consent was obtained from the participants prior to filling online questionnaire in an anonymous way and in a group (pooled) fashion. The study was approved by the ethics committee of Iran University of Medical Sciences (IR.IUMS.REC.1399.002).

\section{Statistical analysis}

Categorical variables were reported as counts and percentages and quantitative variables as means and standard deviations (otherwise mentioned). The relationship between the number of participants and the number of confirmed COVID-19 patients (from national reports) was analyzed by the Spearman correlation coefficient. This part of the assessment has been considered as an ecological study because data were not at individual level.

\section{Results}

The results are based on the reported cases of anosmia or hyposmia in an Iranian population, gathered by an online questionnaire developed by Ear, Nose, Throat and Head and Neck Surgery Research Center of Iran University of Medical Sciences in cooperation with Iran Medical Council from March 12, 2020 (15:20) until March 17, 2020 (10:32).

During this period, 15228 people viewed the online questionnaire, of whom 10249 reported olfactory dysfunction and answered the questions. The following participants 
were excluded from the analysis: 25 participants who did not respond to more than 10 questions (from total of 36 questions), 155 duplicated records that were similar in personal information, and 8 participants in whom the duration of disease was more than 30 days. Thus, the final analysis represented 10069 responders.

Based on demographic data, age distribution ranged from 7 to 78 years $(32.5 \pm 8.6), 71.13 \%$ were female and $81.68 \%$ nonsmoker.

Most responders were from Gilan (51.9\%), Tehran (18.4\%), Mazandaran (6.6\%), Golestan (4.3\%), and Qom $(2.7 \%)$ provinces. Despite a significant difference in the number of responders from multiple geographical areas, the distribution of demographic and clinical variables was homogenous among different provinces of Iran.
The number of cases of anosmia was compared to the official report of patients with COVID-19 in various provinces until March 17, 2020 (Fig. 1), and a significant correlation was found between frequency of anosmia and COVID-19 in various provinces (Spearman Coefficient: 0.87, $\mathrm{P}<0.001$, Fig. 2).

From the clinical point of view, $60.90 \%$ of the responders reported complete loss of sense of smell. The onset of anosmia was sudden in $76.24 \%$, and $80.38 \%$ also reported some decrease in their sense of taste. The clinical findings are summarized in Table 1.

Approximately, $10.55 \%$ of the responders had a history of a trip out of their home town before the onset of their olfactory dysfunction, and about $1.1 \%$ were hospitalized

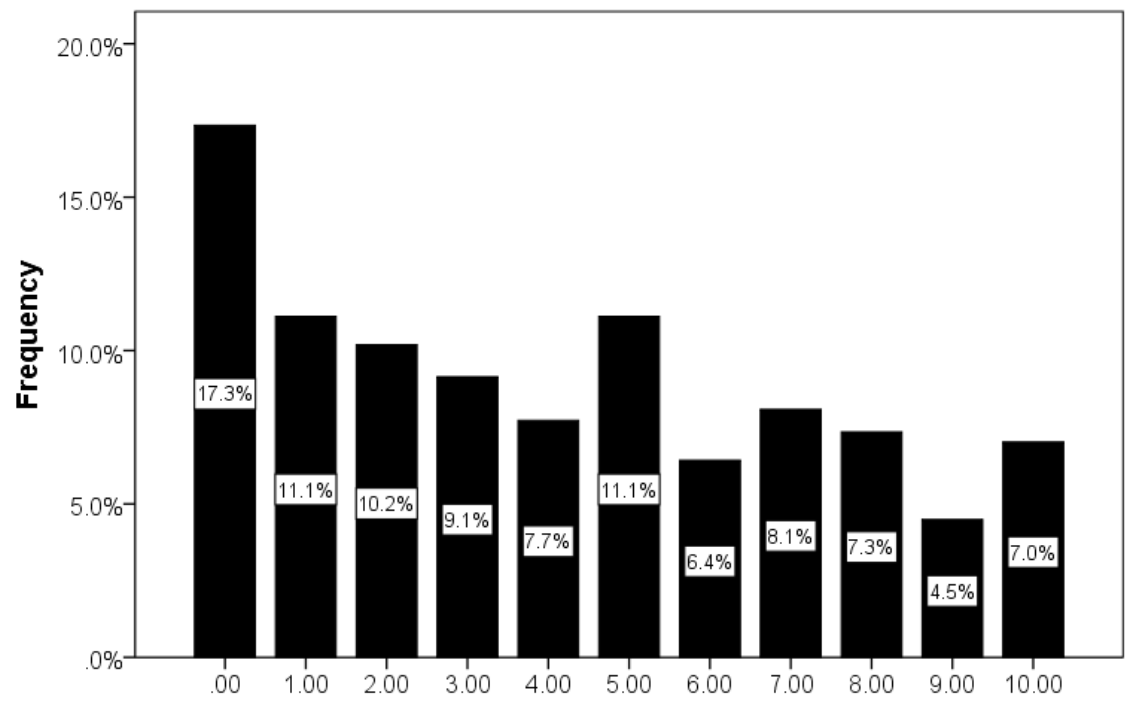

Fig. 1. Numerical scale of smell sensation at the time of filling questionnaire Zero (no sense of smell), 10 (full sense of smell)

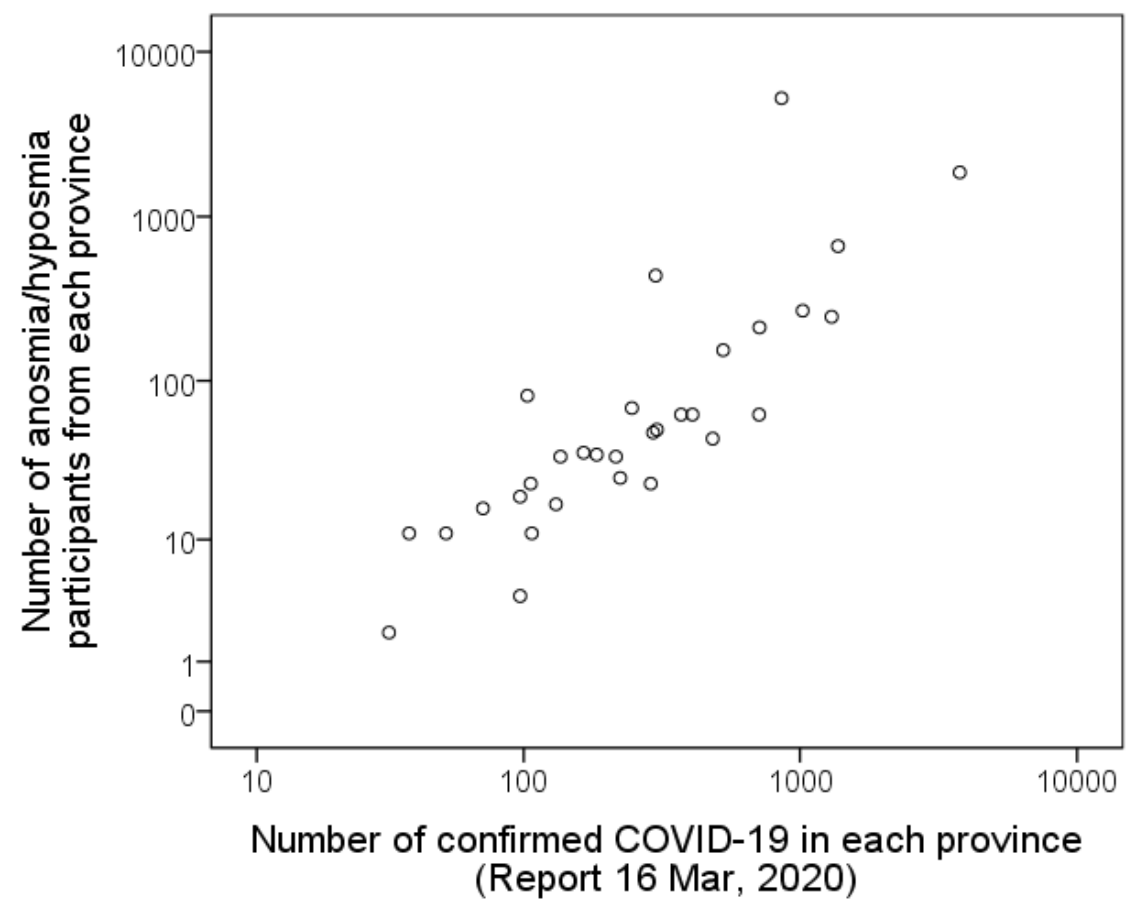

Fig. 2. Correlation between olfactory dysfunction and COVID-19 positivity in different provinces of Iran 


\begin{tabular}{lcc} 
Table 1. Summary of clinical manifestations and past medical history of participants & \\
\hline Clinical manifestation & No. & $\%$ \\
\hline Flu or cold symptoms before anosmia & 7608 & $75.56 \%$ \\
Headache (which needed pain reliever drug) & 4903 & $48.69 \%$ \\
Nasal stiffness & 4400 & $43.70 \%$ \\
Fever and chills & 3764 & $37.38 \%$ \\
Sore throat & 1948 & $19.35 \%$ \\
Prominent cough & 1911 & $18.98 \%$ \\
Orbital (periorbital) pain & 1898 & $18.85 \%$ \\
Facial fullness and sinus pain & 1864 & $18.51 \%$ \\
Rhinorrhea & 1574 & $15.63 \%$ \\
Dyspnea & 1448 & $14.38 \%$ \\
Nasal irritation & 1380 & $13.71 \%$ \\
Parosmia & 1287 & $12.78 \%$ \\
Nasal pruritus & 1083 & $10.76 \%$ \\
Otalgia & 999 & $9.92 \%$ \\
Sneezing frequent & 914 & $9.08 \%$ \\
Purulent nasal discharge & 879 & $8.73 \%$ \\
Cheeks pain & 452 & $4.49 \%$ \\
Unilateral facial palsy & 52 & $0.52 \%$ \\
Past medical history & & \\
History of hypothyroidism & 1077 & $10.70 \%$ \\
History of asthma & 612 & $6.08 \%$ \\
History of hypertension & 428 & $4.25 \%$ \\
Previous sinus surgery or septorhinoplasty & 266 & $2.64 \%$ \\
History of diabetes mellitus & 199 & $1.98 \%$ \\
History of hyperthyroidism & 158 & $1.57 \%$ \\
History of epilepsy & 47 & $0.47 \%$ \\
\hline No. & &
\end{tabular}

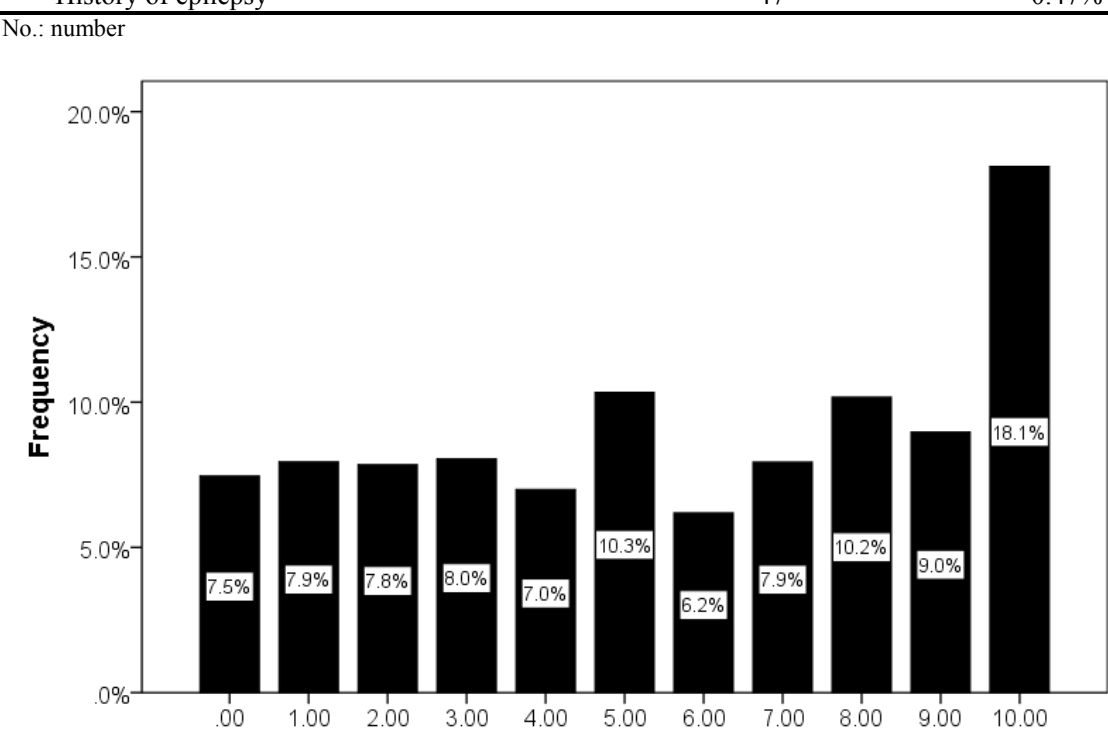

Fig. 3. Numerical Scale of taste sensation at the time of filling questionnaire Zero (no sense of taste), 10 (full sense of taste)

due to recent respiratory problems. From the family point of view, in $12.17 \%$ of the responders, one of the close relatives had a history of severe respiratory disease in recent days, and in $7.35 \%$ at least one of their family members was hospitalized due to respiratory problem. In $48.23 \%$ of responders, at least one of the family members reported a recent history of anosmia. At the time of the response to the questions, the duration of anosmia ranged from 0 (the same day of onset) to 30 days $(11.33 \pm 6.81$, median $=10.00)$.

Use of a numerical scale which coded as 0 (no smell/taste, significantly decreased quality of life) to 10 (full smell/taste, normal quality of life) showed that a significant number of patients still had smell and taste problems at the time of responding to the questionnaire; however, some improvements could also be noted (Figs. 1 and
3). The quality of life was obviously affected due to olfactory dysfunction (Fig. 4).

\section{Discussion}

During March 2020, the number of patients with olfactory dysfunction was increased in different provinces of Iran, concurrent with COVID-19 epidemic. Thus, we decided to assess the prevalence of this olfactory dysfunction disorder by an online questionnaire in this population-based study. The results of this study showed a widespread olfactory dysfunction all over the country. Also, a significant linear correlation between prevalence of COVID-19 and olfactory impairment that was published online on March 27, 2020 (11).

The recent outbreak of olfactory dysfunction following 


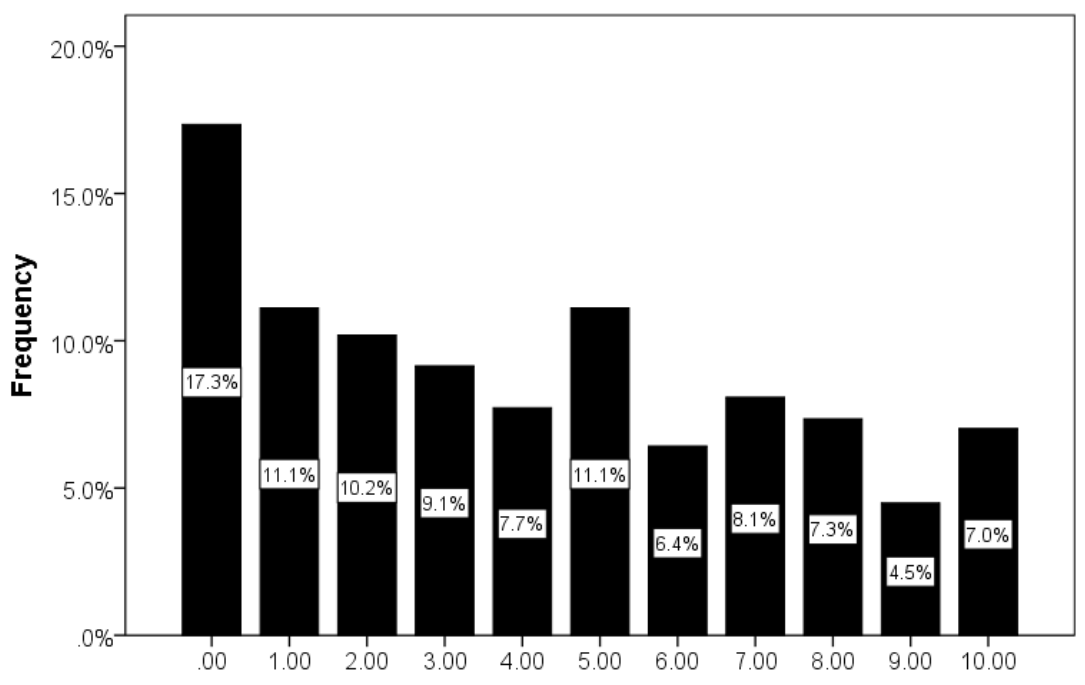

Fig. 4. Numerical Scale of quality of life at the time of filling questionnaire. Zero (significantly decreased quality of life), 10 (normal quality of life)

flu-like symptoms $(75 \%)$, higher incidence in women $(71 \%)$, and a high incidence of anosmia in family members $(48.23 \%)$ are suggestive of a postviral epidemic olfactory dysfunction in Iran. Although our study could not establish that COVID-19 was the definite cause of olfactory dysfunction, there are no other recent viral epidemics in Iran except COVID-19. Thus, as the COVID-19 pandemic rises in parallel with olfactory dysfunction, it can be suggested that these 2 are intimately linked. However, later studies in patients with definite diagnosis of COVID-19 showed that olfactory dysfunction had a considerable prevalence in these patients (12-14). For instance, a study on 417 COVID-19 patients was performed by Lechien et al and showed that $79.6 \%$ of COVID-19 patients had olfactory dysfunction (15). In addition, a study by Menni et al found that $59 \%$ of COVID-19 positive patients had loss of smell and taste as compared to $18 \%$ of COVID-19 negative patients (16). Moreover, Moein et al showed that $98 \%$ of patients with COVID-19 exhibited some smell dysfunction, of them 58\% were either anosmic or severely microsmic (17). Based on these reports, Menni et al and Kaye et al suggested that olfactory dysfunction should be considered as a manifestation of COVID-19 $(16,18)$.

Common COVID-19 symptoms, such as fever, cough and dyspnea, were less common in participants with olfactory dysfunction compliant $(87.9 \%$ vs $37.38 \%, 67.7 \%$ vs $18.98 \%$ and $18.6 \%$ vs $14.38 \%$, respectively) and only about $1.1 \%$ of the study population was hospitalized due to respiratory problems recently.

In 2007, Suzuki et al detected Rhinovirus, Coronavirus, Parainfluenza virus, and Epstein-Barr virus in the nasal secretion of postviral olfactory disorders patients for the first time. Also, they suggested that the Rhinoviruses can target olfactory impairment through mechanisms other than nasal obstruction and that Rhinoviruses could induce different time courses of olfactory dysfunction (19).

Although the exact pathophysiology of postviral anosmia is unclear, injury may happen at the level of the neuroepithelium of olfactory receptor cells in the nasal roof or in central olfactory routs. However, many studies have focused on the neuroepithelial changes in patients with postviral olfactory dysfunction because of the accessibility of the olfactory cleft. Various animal studies explained that different viruses could damage central olfactory routs and other regions of the brain (20-23). It has been found that $36.4 \%$ of patients with COVID-19 had a variety of neurological symptoms involving central and peripheral nervous system. In addition, central nervous system findings were a more significant form of neurological damage in patients with COVID-19. (10) Thus as SARS-CoV-2 can reach the brain through the cribriform plate (24), anosmia or hyposmia in an uncomplicated COVID-19 case may also need to be considered.

There are some limitations to our study. As our data were collected via an online self-reported questionnaire, it was limited to the population who was literate and had access to the internet, personal computers or smart-phones, and were active in social networks. Another limitation of this study is that the participants had not been evaluated for SARSCoV-2 test. As this was an online survey, the main goal was to confirm the outbreak of olfactory dysfunction during the COVID-19 epidemic.

\section{Conclusion}

We encountered an outbreak of olfactory dysfunction in Iran during the COVID-19 epidemic, which correlates with the number of patients infected with COVID-19 all over the country. As olfactory dysfunction can affect the quality of life in affected patients, it needs to be assessed worldwide by further clinical studies to find pathogenesis, prognosis, and any correlation between disease severity and olfactory dysfunction. Also, we recommend that sudden onset of anosmia be considered in routine work-up and screening for COVID-19 infection. 


\section{Acknowledgements}

Authors wish to thank patients for their participation and kind cooperation.

\section{Conflict of Interests}

The authors declare that they have no competing interests.

\section{References}

1. Hummel T, Whitcroft KL, Andrews P, Altundag A, Cinghi C, Costanzo RM, et al. Position paper on olfactory dysfunction. Rhinology. 2016;56(1):1-30.

2. Welge-Lüssen A. Re-establishment of olfactory and taste functions. GMS Curr Top Otorhinolaryngol Head Neck Surg. 2005;4:Doc06Doc.

3. Hummel T, Landis BN, Hüttenbrink KB. Smell and taste disorders GMS Curr Top Otorhinolaryngol Head Neck Surg. 2011;10:Doc04Doc.

4. Jafek BW, Murrow B, Michaels R, Restrepo D, Linschoten M. Biopsies of human olfactory epithelium. Chem Senses. 2002;27(7):623-8.

5. World Health Organization. March 2020.

6. Li G, De Clercq E. Therapeutic options for the 2019 novel coronavirus (2019-nCoV). Nat Rev Drug Discov. 2020;19(3):149-50.

7. WHO. Coronavirus disease 2019 (COVID-19): situation report, 2020;93.

8. Rothan H, Siddappa N. The epidemiology and pathogenesis of coronavirus disease (COVID-19) outbreak. J Autoimmun. 2020;109:102433.

9. Chan KW, Wong VT, Tang SCW. COVID-19: An Update on the Epidemiological, Clinical, Preventive and Therapeutic Evidence and Guidelines of Integrative Chinese-Western Medicine for the Management of 2019 Novel Coronavirus Disease. Am J Chin Med. 2020:1-26.

10. Mao L, Wang M, Chen S, He Q, Chang J, Hong C, et al. Neurological Manifestations of Hospitalized Patients with COVID-19 in Wuhan, China: a retrospective case series study. JAMA Neurol. 2020.

11.Bagheri SHR, Asghari AM, Farhadi M, Shamshiri AR, Kabir A, Kamrava SK, et al. Coincidence of COVID-19 epidemic and olfactory dysfunction outbreak. medRxiv. 2020.

12. Yan CH, Faraji F, Prajapati DP, Boone CE, DeConde AS. Association of chemosensory dysfunction and COVID-19 in patients presenting with influenza-like symptoms. Int Forum Allergy Rhinol. 2020.

13. Soler ZM, Patel ZM, Turner JH, Holbrook EH. A primer on viralassociated olfactory loss in the era of COVID-19. Int Forum Allergy Rhinol. 2020.

14. Walker A, Hopkins C, Surda P. The use of google trends to investigate the loss of smell related searches during COVID-19 outbreak. Int Forum Allergy Rhinol. 2020

15. Lechien JR, Chiesa-Estomba CM, De Siati DR, Horoi M, Le Bon SD, Rodriguez A, et al. Olfactory and gustatory dysfunctions as a clinical presentation of mild-to-moderate forms of the coronavirus disease (COVID-19): a multicenter European study. Eur Arch Otorhinolaryngol. 2020.

16. Menni C, Valdes A, Freydin MB, Ganesh S, El-Sayed Moustafa J, Visconti A, et al. Loss of smell and taste in combination with other symptoms is a strong predictor of COVID-19 infection. medRxiv. 2020.

17. Moein ST, Hashemian SMR, Mansourafshar B, Khorram-Tousi A, Tabarsi P, Doty RL. Smell dysfunction: a biomarker for COVID-19. Int Forum Allergy Rhinol. 2020.

18. Rachael Kaye, CW David Chang, Ken Kazahaya, Jean Brereton, Denneny J. COVID-19 Anosmia Reporting Tool: Initial Findings. Otolaryng Head Neck. 2020.

19. Suzuki M, Saito K, Min WP, Vladau C, Toida K, Itoh H, et al. Identification of viruses in patients with postviral olfactory dysfunction. Laryngoscope. 2007;117(2):272-7.

20. Seiden AM. Postviral olfactory loss. Otolaryng Clin N Am. 2004;37(6):1159-66.

21. Duncan HJ. Postviral olfactory loss; in Seiden AM (ed): Taste and Smell Disorders. 1997:72-8.

22. Mohammed AK, Magnusson O, Maehlen J, Fonnum F, Norrby E, Schultzberg M, et al. Behavioural deficits and serotonin depletion in

$6 \quad$ http://mjiri.iums.ac.ir Med J Islam Repub Iran. 2020 (15 Jun); 34:62. adult rats after transient infant nasal viral infection. Neuroscience. 1990;35(2):355-63.

23. Perlman S, Evans G, Afifi A. Effect of olfactory bulb ablation on spread of a neurotropic coronavirus into the mouse brain. J Exp Med. 1990;172(4):1127-32.

24. Baig AM, Khaleeq A, Ali U, Syeda H. Evidence of the COVID-19 Virus Targeting the CNS: Tissue Distribution, Host-Virus Interaction, and Proposed Neurotropic Mechanisms. ACS Chem Neurosci. 2020;11(7):995-8 


\begin{tabular}{|c|c|c|c|}
\hline $\mathrm{N}$ & Question & \multicolumn{2}{|c|}{ Response Options } \\
\hline 1 & How did the onset of your olfactory dysfunction happen? & Slowly & Suddenly \\
\hline 2 & Did you have olfactory dysfunction along with gustatory dysfunction? & Yes & No \\
\hline 3 & $\begin{array}{l}\text { Have you had any flu or cold symptoms (fever, rhinorrhea, cough, and sore } \\
\text { throat) before anosmia? }\end{array}$ & Yes & No \\
\hline 4 & Have you had a severe headache that needed a pain reliever drug? & Yes & No \\
\hline $5-7$ & $\begin{array}{l}\text { Have you had fever and chills/ severe cough/ dyspnea before or during the olfac- } \\
\text { tory dysfunction? }\end{array}$ & Yes & No \\
\hline 8 & Did you smell the natural odors as unpleasant smells? & Yes & No \\
\hline 9 & Rate your ability to smell during the first days of your olfactory dysfunction. & \multicolumn{2}{|c|}{ Line scale $(0-10)$} \\
\hline 10 & Rate your ability to taste during the first days of your olfactory dysfunction. & \multicolumn{2}{|c|}{ Line scale $(0-10)$} \\
\hline 11 & How many days have you had olfactory dysfunction? & \multicolumn{2}{|c|}{$0-30$} \\
\hline 12 & Rate your ability to smell right now (at the time of filling this questionnaire). & \multicolumn{2}{|c|}{ Line scale $(0-10)$} \\
\hline 13 & Rate your ability to taste right now (at the time of filling this questionnaire). & \multicolumn{2}{|c|}{ Line scale $(0-10)$} \\
\hline 14 & Did you use any medication for your olfactory dysfunction? & Yes & No \\
\hline 15 & How was the pattern of your olfactory dysfunction? & Always/consistency & Sometimes/inconsistency \\
\hline 16 & Did you get any nasal surgery such as previous sinus surgery or septorhinoplasty? & Yes & No \\
\hline 17 & Rate the negative effect of your olfactory dysfunction on your quality of life. & \multicolumn{2}{|c|}{ Line scale $(0-10)$} \\
\hline \multirow[t]{13}{*}{18} & Which ones did you have along with your olfactory dysfunction? & \multirow[t]{13}{*}{ Yes } & \multirow[t]{13}{*}{ No } \\
\hline & Nasal stiffness & & \\
\hline & Nasal pruritus & & \\
\hline & Nasal irritation & & \\
\hline & Sneezing (frequent) & & \\
\hline & Rhinorrhea & & \\
\hline & Purulent nasal discharge & & \\
\hline & Cheeks pain & & \\
\hline & Facial fullness and sinus pain & & \\
\hline & Orbital (periorbital) pain & & \\
\hline & Sore throat & & \\
\hline & Otalgia & & \\
\hline & Unilateral facial palsy & & \\
\hline
\end{tabular}

